\title{
Optimization and Customer Utilities under Dynamic Lead Time Quotation in an $M / M$ Type Base Stock System
}

\author{
Koichi Nakade and Hiroki Niwa \\ Department of Architecture, Civil Engineering and Industrial Management Engineering, Nagoya Institute of Technology, \\ Gokiso-cho, Showa-ku, Nagoya 466-8555, Japan \\ Correspondence should be addressed to Koichi Nakade; nakade@nitech.ac.jp
}

Received 23 February 2017; Revised 10 April 2017; Accepted 2 May 2017; Published 25 May 2017

Academic Editor: Huanqing Wang

Copyright (C) 2017 Koichi Nakade and Hiroki Niwa. This is an open access article distributed under the Creative Commons Attribution License, which permits unrestricted use, distribution, and reproduction in any medium, provided the original work is properly cited.

\begin{abstract}
In a manufacturing and inventory system, information on production and order lead time helps consumers' decision whether they receive finished products or not by considering their own impatience on waiting time. In Savaşaneril et al. (2010), the optimal dynamic lead time quotation policy in a one-stage production and inventory system with a base stock policy for maximizing the system's profit and its properties are discussed. In this system, each arriving customer decides whether he/she enters the system based on the quoted lead time informed by the system. On the other hand, the customer's utility may be small under the optimal quoted lead time policy because the actual lead time may be longer than the quoted lead time. We use a utility function with respect to benefit of receiving products and waiting time and propose several kinds of heuristic lead time quotation policies. These are compared with optimal policies with respect to both profits and customer's utilities. Through numerical examples some kinds of heuristic policies have better expected utilities of customers than the optimal quoted lead time policy maximizing system's profits.
\end{abstract}

\section{Introduction}

In manufacturing systems the production and inventory control must be appropriate to reduce the production cost. Information on production such as advance demand, amounts of work-in-process and finished products, and machine's failure is important to the control. For customers buying products, the information of order lead time is important to decide whether he/she buys a product or not. In addition, the consumer will be unsatisfied when the actual lead time is greater than the informed lead time. The quotation of lead time causes the actual number of customers to vary because the long quoted lead time leads to decrease of actual demand, and thus appropriate information makes the profit of the system increase.

Recently, the information on inventory or lead time to customers is discussed. For example, Duenyas and Hopp [1] develop a dynamic lead time quotation problem in a make-to-order system as an $M / M / 1$ queue using an MDP formulation. Ata and Olsen [2] consider a make-to-order system where customers are dynamically quoted lead times.
They recommend quotation policies for convex, concave, and convex-concave delay cost functions. Kapuscinski and Tayur [3] consider two classes of customers, where the high priority customers bring more profit to the system but delay for them leads to the higher penalty on the system. Wu et al. [4] consider a newsvendor problem with information of price and quoted lead time and determine optimal amounts of orders, prices, and quoted lead time. In Selçuk [5] a cost effective dynamic lead time quotation procedure in a single-stage controlled manufacturing system is considered and guidelines are discussed for setting the number of kanbans and the frequency of updating lead time. Slotnick [6] discusses an optimal lead time quotation policy when reputation of firm affects whether each consumer orders products or balks and discusses the relationships among order size, reputation, lead time decision, and so on. In addition, Hafizoğlu et al. [7] consider price and lead time decisions in a make-tostock system with contract and spot customers under Poisson arrivals and exponential service times. The optima policy on price and lead time is characterized. Zhao et al. [8] consider the make-to-order manufacturing system which gives two 
lead time and price quotation strategies, one of which offers a single lead time and price, and the other gives the menu of pairs of lead time and prices. They discuss the better strategies under the price-sensitive and lead time sensitive consumers. The other lead time quotation and decision models are found in Keskinocak et al. [9], Ata [10], Charnsirisakskul et al. [11], Chaharsooghi et al. [12], and Xiao et al. [13].

In Savaşaneril et al. [14], the optimal lead time quotation in an $M / M / 1$ base stock inventory queue for maximizing the system's profit is discussed. In this system, if the lead time quoted to the arriving customer is long, the probability that the customer enters the system and receives service becomes small. In their paper, the model is formulated as a Markov decision process and the property of the optimal lead time quotation policy is discussed.

Literature on optimal quotation polices focuses on the optimal lead quotation policies for the system. Under such optimal lead time quotation policies, however, the exact lead time information is not quoted to customers. One reason is that the production process is under uncertainness because of failure or repair of the machine, and thus actual production time is stochastic. The other and important reason is that, under the optimal lead time policy, the system manager may not give the mean of actual lead time, even if the delay cost for quoted lead time is considered. Thus, when this optimal policy is applied, the customer's satisfaction may be small, because he/she leaves the system by quotation of long lead time when the actual lead time is small and his/her actual waiting time for items may be longer than quoted lead time because of the setting of shorter quoted lead time than the actual lead time. Thus, for the system manager, it is important to maximize not only the profit of the system but also customer's satisfaction. Most of researches in literature, however, do not discuss this kind of satisfaction of actual customers deeply.

As one of the customer's utility on waiting time, the effect of delay information on the customer's satisfaction is formulated in Guo and Zipkin $[15,16]$. In these papers, the utility function of each customer is defined, which shows the degree of his/her satisfaction on waiting time in a queue. It includes the stochastic parameter representing his/her impatience for waiting time, and if the value of this parameter is high, then he/she is impatient of waiting. They defined several types of delay information and their effects are discussed theoretically and numerically. In Nakade and Niwa [17], an $M / M / 1$ base stock manufacturing-inventory system is developed, but the evaluation of the utility function is inappropriate and thus the relationship between the utility and average cost is unclear. In fact, the utility function itself only includes the waiting time cost, but the evaluation of the utility as performance measure includes the delay cost for quoted lead time, which is confusing.

In this paper, the effect by the lead time quotation in an $M / M / 1$ base stock manufacturing-inventory queue into the customer's utility function is discussed. Poisson arrivals are popular because it is well known that the arrival process from large population with small probability that each person will arrive at a system approximately forms a Poisson process (e.g., see section 5.2 of Pinsky and Karlin [18]). The utility function is based on the definition in Guo and Zipkin [15], and if his/her utility is negative under the lead time information then he/she leaves the system without receiving an item and otherwise enters the system and receives it after possible waiting time. The model is formulated into a Markov decision process. Its optimal lead time quotation policy is derived, and several heuristic policies such as linear, convex, and concave lead time quotation policies are analyzed by birth and death processes numerically. Numerical results are modified and extended from Nakade and Niwa [17]. In comparison between profits and utilities, the performance measure of utility does not include the delay for quoted lead time. In addition, the relationship among expected reward, the inventory cost, and the delay cost for quoted lead time under optimal policy is discussed for each base stock and fixed delay cost. The numerical examples show that the optimal lead time quotation policy for maximizing system's average profit has low customer's utility, and the other simple heuristic quotation policy leads to the greater expected values of customers' utility, although it has a bit smaller system's profit than the optimal policy. In particular, some lead time quotation policy has both more system profit and much customer's satisfaction in comparison with the optimal lead time quotation policy with the greater number of base stocks.

The organization of this paper is as follows. In Section 2, a lead time quotation model and a utility function in a manufacturing-inventory system with base stocks are defined. In Section 3, an average cost under a given quotation policy is determined and the optimization problem in this system is formulated as a Markov decision process. The expected utility is also derived. Numerical experiments for developing properties of optimal policies and other heuristic policies are given in Section 4, and the concluding remarks are given in Section 5.

\section{Lead Time Quotation Model}

2.1. Model Description. A manufacturing-inventory system with a single process is considered. Customers arrive in a Poisson process with rate $\lambda$, and the production time has an exponential distribution with rate $\mu$. The processing time is mutually independent among products and also independent of the arrival process. A base stock policy with base stock level $s$ is applied in this system. Figure 1 illustrates the model.

The state of the system is inventory position $i$, and if $i<0$ then the system has $(-i)$ products as inventory, and if $i>0$ then $i$ denotes the number of waiting customers for products. When $i=0$, the system has no finished products in inventory and no waiting customers.

When a customer arrives at the system whose state is $i$, the system quotes him/her lead time $d_{i}$ which is an estimated lead time and is based on the inventory level $i$. After the arriving customer receives this information, he/she determines whether he/she enters the system or not. The decision of each customer follows his/her own parameter $\theta$, which represents impatience on the waiting. This parameter $\theta$ has a distribution function $H(\theta)$ on $[0,1]$, whose density is $h(\theta)$. Details on how customers decide whether they buy products or not are discussed in Section 2.2. 


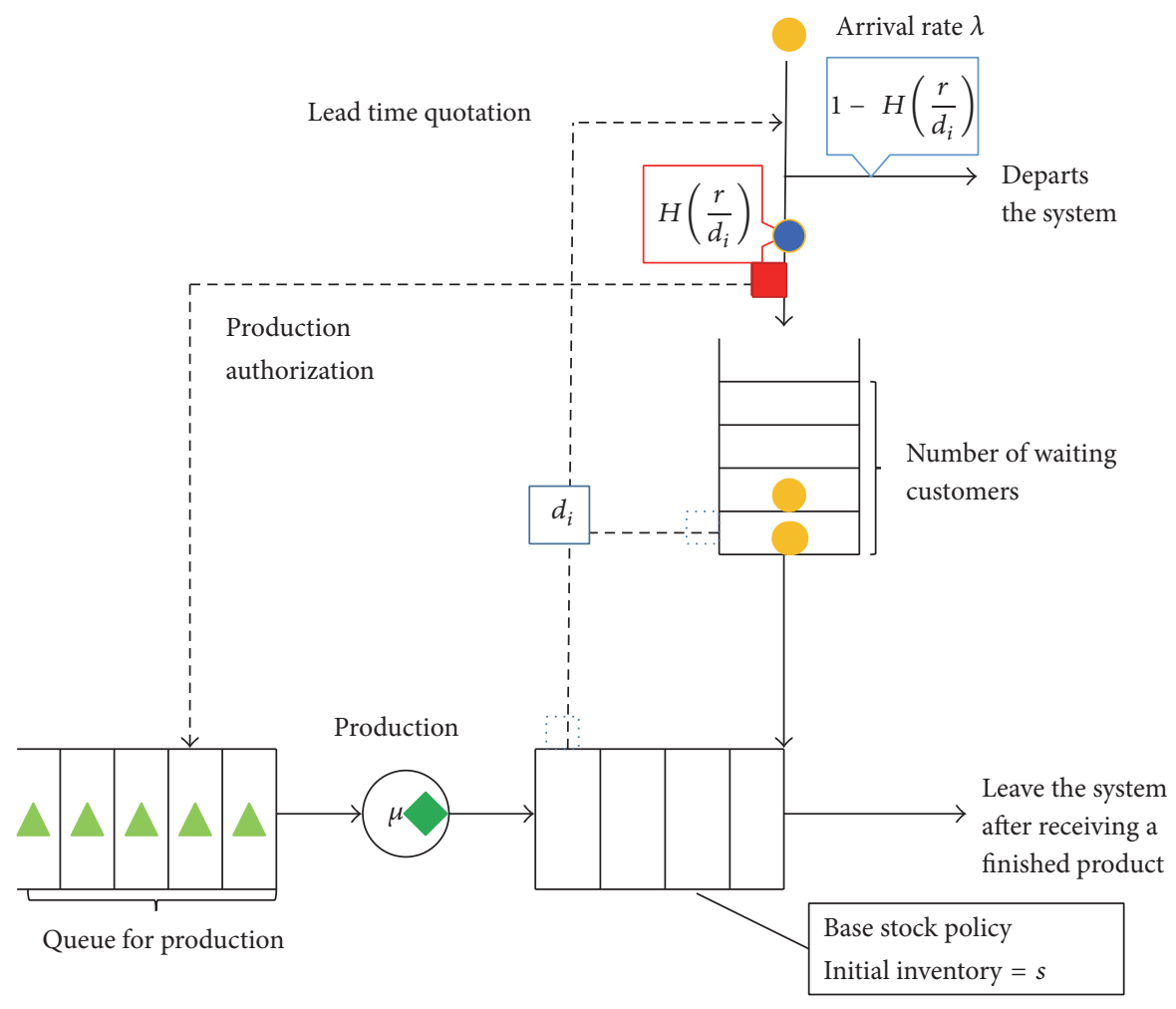

FIGURE 1: Base stock system with lead time quotation.

When he/she enters the system, one item is ordered under a base stock policy. If there is a finished product, then he/she receives it immediately and leaves the system. It is assumed that the quoted lead time is discrete and in a set of $\{0,0.05,0.10, \ldots\}$. The service is based on first come, first served basis.

When a customer enters the system, the system receives reward $R$. An inventory cost rate $h$ for each finished product in inventory is incurred. In addition, when the delay happens against quoted lead time $d_{i}$, the fixed delay cost $c$ and the delay cost rate $l$, which are proportional to delay time, are also incurred for the system.

We use the following notations.

$d_{i}$ : quoted lead time when the level is $i$

$$
d_{i} \in\{0,0.05,0.10, \ldots\}
$$

$f(d)$ : the probability that the customer to whom the planned lead time $d$ is quoted enters the system, where it is assumed that $f(0)=1$ and $0 \leq f(d) \leq 1$.

$d_{\max }$ : the minimal value $d \in\{0,0.05,0.10, \ldots\}$ which satisfies $f(d)=0$,

$d_{\text {min }}$ : the maximal value $d \in\{0,0.05,0.10, \ldots\}$ which satisfies $f(d)=1$.

When $c=0$, the model coincides with one in Savaşaneril et al. [14] except that they do not consider the distribution of utility function of customers. In the next section, the utility function is defined.
2.2. Utility. In this paper, we assume that the utility function of each customer, when the impatience degree is $\theta$ and quoted lead time is $d_{i}$, is given by

$$
U\left(\theta, d_{i}\right)=r-\theta d_{i}
$$

where $r$ is the value of product which each customer will receive and $r$ is assumed positive. The utility function is based on Guo and Zipkin [15]. The linear sensitivity of the utility function may be extended to nonlinear cases, but it is assumed to explain the model more easily by treating a lead time quotation policy as a simple threshold policy. The arriving customer decides whether he enters the system or not by the sign of this value. That is, if $U\left(\theta, d_{i}\right) \geq 0$ then the customer enters the system, and otherwise he leaves system without entrance into the system. For state $i$ which an entering customer finds, if $i<0$, when the customer enters the system there is no waiting time, and thus his actual utility is $r$. If $i \geq 0$ the customer waits for receiving product with information $d_{i}$. Thus, a sequence $\left\{d_{i} ; i=0,1,2, \ldots\right\}$ is system's lead time quotation policy which controls the entrance of customers.

We define $\theta_{i}=r / d_{i}$ for a given policy $\left\{d_{i} ; i=0,1,2, \ldots\right\}$. From (2), a customer enters the system when the state is $i$ if and only if the random parameter $\theta$ of the customer is smaller than or equal to $\theta_{i}$ under this policy. That is, when the lead time $d_{i}$ is quoted, the customer will enter the system with probability $f\left(d_{i}\right)=H\left(r / d_{i}\right)$. 


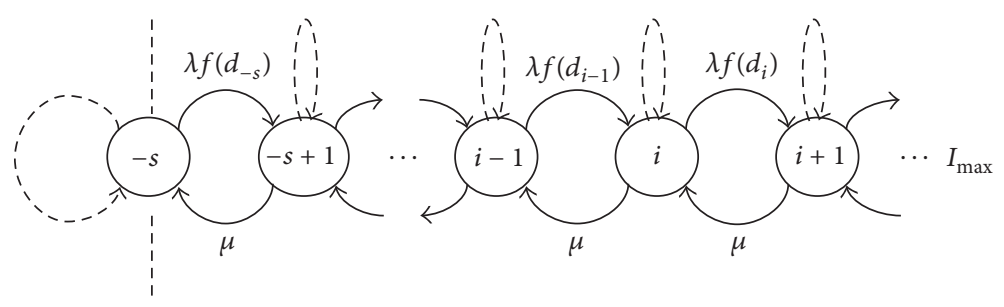

FIGURE 2: Transition diagram.

We denote the maximal $\theta$ satisfying $H(\theta)=0$ by $\theta_{L}$, which is assumed to be positive. Since $d_{\max }(<+\infty)$ denotes the minimal $d$ satisfying $f\left(d_{i}\right)=0$, we have

$$
d_{\max }=\min \left(d: \frac{r}{\theta_{L}} \leq d\right)=\frac{r}{\theta_{L}} .
$$

Let $I=\left\{-s,-s+1,-s+2, \ldots, 0,1, \ldots, I_{\max }\right\}$ denote the set of possible inventory positions. When $d_{i}=d_{\max }$, the customer will not enter the system, and thus the inventory position will not become $i+1$ or more. Thus, $I_{\max }$, which is the maximal value of possible states, is an inventory position in which the decision $d_{i}=d_{\max }$ is taken.

In this paper, the system is assumed to be in steady state, that is, reputation of customers against the manufacturer is stable under the fixed lead time quotation policy. Note that when state $i$ is negative, the arriving customer can receive a finished product immediately, and thus $d_{i}$ is set as 0 .

2.3. Stationary Probabilities. When the quotation policy $\vec{d}=$ $\left(d_{-s}, d_{-s+1}, \ldots, d_{I_{\max }}\right)$ is decided, the process of the state of system becomes a birth and death process with parameters $\lambda f\left(d_{i}\right)$ and $\mu$, as shown in Figure 2. Thus, the stationary probability that the inventory position is $i$ is given by

$$
p_{i}(\vec{d})=\left(\frac{\lambda}{\mu}\right)^{i+s} f\left(d_{i-1}\right) f\left(d_{i-2}\right) \cdots f\left(d_{-s}\right) p_{-s}(\vec{d}),
$$

$i \in I$,

where

$$
\begin{aligned}
p_{-s}(\vec{d}) & \\
= & \frac{1}{1+\sum_{i=-s+1}^{I_{\max }}(\lambda / \mu)^{i+s} f\left(d_{i-1}\right) f\left(d_{i-2}\right) \cdots f\left(d_{-s}\right)} .
\end{aligned}
$$

\section{Profit and Utility}

3.1. Average Profit. Under policy $\vec{d}=\left(d_{-s}, d_{-s+1}, \ldots, d_{I_{\max }}\right)$, the expected profit obtained from customers per unit time is given by

$$
R(\vec{d})=R \times \lambda \sum_{i=-s}^{I_{\max }} p_{i}(\vec{d}) f\left(d_{i}\right)
$$

The expected inventory cost per unit time, $S(\vec{d})$, is given by

$$
S(\vec{d})=h \sum_{i=-s}^{-1}(-i) p_{i}(\vec{d})
$$

The probability that the delay happens when the lead time $d_{i}$ is quoted to the customer in state $i$ is denoted by $C_{i}\left(d_{i}\right)$. When $i<0$, the customer receives a product immediately and thus $C_{i}\left(d_{i}\right)=0$. Since the production time follows the exponential distribution with rate $\mu$, we have

$$
C_{i}\left(d_{i}\right)= \begin{cases}\sum_{j=0}^{i} \frac{\left(\mu d_{i}\right)^{j}}{j !} e^{-\mu d_{i}}: & i=0,1,2, \ldots \\ 0: & i<0 .\end{cases}
$$

Thus, the average expected fixed delay cost for quoted lead time is given by

$$
D(\vec{d})=c\left\{\lambda \sum_{i=0}^{I_{\max }} p_{i}(\vec{d}) f\left(d_{i}\right) \times \sum_{j=0}^{i} \frac{\left(\mu d_{i}\right)^{j}}{j !} e^{-\mu d_{i}}\right\} .
$$

In the same way, the expected delay for the customer entering in state $i$ is given by

$$
\begin{aligned}
& L_{i}\left(d_{i}\right) \\
& \quad= \begin{cases}\int_{d_{i}}^{\infty}\left(x-d_{i}\right) \frac{\mu\left(\mu d_{i}\right)^{i}}{i !} e^{-\mu x} d x & i=0,1,2, \ldots \\
0 & i<0 .\end{cases}
\end{aligned}
$$

Since

$$
\begin{aligned}
L_{i}\left(d_{i}\right) & =\int_{d_{i}}^{\infty} \frac{\mu^{i+1} x^{i+1}}{i !} e^{-\mu x} d x-d_{i} \int_{d_{i}}^{\infty} \frac{\mu^{i+1} x^{i}}{i !} e^{-\mu x} d x \\
& =\frac{i+1}{\mu} e^{-\mu d_{i}}+d_{i} \sum_{j=0}^{i}(i-j) \frac{\left(\mu d_{i}\right)^{j}}{(j+1) !} e^{-\mu d_{i}},
\end{aligned}
$$


the expected delay cost on quoted lead time is given by

$$
\begin{aligned}
& T(\vec{d})=l\left\{\lambda \sum_{i=0}^{I_{\max }} p_{i}(\vec{d}) f\left(d_{i}\right)\right. \\
& \left.\quad \times\left(\frac{i+1}{\mu} e^{-\mu d_{i}}+d_{i} \sum_{j=0}^{i}(i-j) \frac{\left(\mu d_{i}\right)^{j}}{(j+1) !} e^{-\mu d_{i}}\right)\right\} .
\end{aligned}
$$

Thus, the average expected profit of the system over an infinite horizon under policy $\vec{d}$ is given by

$$
\begin{gathered}
G(\vec{d})=R(\vec{d})-S(\vec{d})-D(\vec{d})-T(\vec{d})=R \\
\times \lambda \sum_{i=-s}^{I_{\max }} p_{i}(\vec{d}) f\left(d_{i}\right)-h \sum_{i=-s}^{-1}(-i) p_{i}(\vec{d})
\end{gathered}
$$

$$
\begin{aligned}
& -c\left\{\lambda \sum_{i=0}^{I_{\max }} p_{i}(\vec{d}) f\left(d_{i}\right) \times \sum_{j=0}^{i} \frac{\left(\mu d_{i}\right)^{j}}{j !} e^{-\mu d_{i}}\right\} \\
& -l\left\{\lambda \sum_{i=0}^{I_{\max }} p_{i}(\vec{d}) f\left(d_{i}\right)\right. \\
& \left.\times\left(\frac{i+1}{\mu} e^{-\mu d_{i}}+d_{i} \sum_{j=0}^{i}(i-j) \frac{\left(\mu d_{i}\right)^{j}}{(j+1) !} e^{-\mu d_{i}}\right)\right\} .
\end{aligned}
$$

Here, the model is formulated as a Markov decision process to derive optimal planned lead time $\vec{d}^{*}=\left\{d_{i}^{*}\right\}$ which maximizes the average reward $G(\vec{d})$. By uniformization technique, the model can be formulated into a discrete-time model with the optimal average reward $g$ and relative reward $v^{*}(i)$ under optimal policy, which satisfies

$$
\begin{aligned}
& g+v^{*}(i) \\
& =\max _{d_{i}}\left\{\begin{array}{l}
-\frac{h i^{-}}{\lambda+\mu}+\frac{\lambda}{\lambda+\mu} f\left(d_{i}\right)\left(R-c C_{i}\left(d_{i}\right)-l L_{i}\left(d_{i}\right)+v^{*}(i+1)\right)+\frac{\lambda}{\lambda+\mu}\left(1-f\left(d_{i}\right)\right) v^{*}(i)+\frac{\mu}{\lambda+\mu} v^{*}(i), \quad(i=-s), \\
-\frac{h i^{-}}{\lambda+\mu}+\frac{\lambda}{\lambda+\mu} f\left(d_{i}\right)\left(R-c C_{i}\left(d_{i}\right)-l L_{i}\left(d_{i}\right)+v^{*}(i+1)\right)+\frac{\lambda}{\lambda+\mu}\left(1-f\left(d_{i}\right)\right) v^{*}(i)+\frac{\mu}{\lambda+\mu} v^{*}(i-1), \quad(i>-s) .
\end{array}\right.
\end{aligned}
$$

Here $i^{-}=\max \{-i, 0\}$. Then $d_{i}^{*}$ maximizes the right hand side of this equation, and the optimal average reward is $G\left(\vec{d}^{*}\right)=$ $(\lambda+\mu) g$. The optimal policy can be solved by the well-known policy iteration method, because for any quotation policy the inventory position can return to $-s$ with probability one (see Puterman [19]).

3.2. Expected Utility. When a customer enters based on the utility function $U\left(\theta, d_{i}\right)$ which is given in (2), its actual utility which the customer receives is different from the value of this utility function, because the delay of the actual lead time compared with quoted lead time may happen. The actual expected utility of entering customer with parameter $\theta$ in state $i$, denoted by $u\left(\theta, d_{i}\right)$, is given by

$$
u\left(\theta, d_{i}\right)=r-\theta \frac{i+1}{\mu}
$$

for $i \geq 0$. Note that $u\left(\theta, d_{i}\right)=r$ when $i<0$. The expected utility of each customer under quotation lead time policy $\vec{d}$ is given by

$$
\begin{aligned}
u(\vec{d})= & \sum_{i=-s}^{-1} p_{i}(\vec{d}) r \\
& +\sum_{i=0}^{I_{\max }} p_{i}(\vec{d}) \int_{\theta_{L}}^{\theta_{i}}\left(r-\phi \frac{i+1}{\mu}\right) h(\phi) d \phi,
\end{aligned}
$$

where $\theta_{i}=r / d_{i}$.

\section{Numerical Experiments}

Given parameters $\lambda, \mu, R, H(\theta), h, l, c, f(d), r$, and $\theta_{L}$, we derive the optimal policy $d_{i}^{*}$ maximizing the average profit of the system for each fixed $s$, and then the optimal amount of base stock level $s^{*}$ is derived. Then several linear lead time policies are proposed, where linear lead time policy means that the quoted lead time is proportional to the inventory size. We discuss these linear policies with optimal policies regarding both profit of the system and the utility of customers. Convex and concave type lead time quotation policies are also introduced.

The parameters used in numerical experiments are set as follows:

$$
\begin{aligned}
\lambda & =0.6, \\
\mu & =1.0, \\
h & =0.5, \\
c & =1.0, \\
l & =1.0, \\
r & =1.0, \\
R & =10, \\
\theta_{L} & =0.25, \\
d & \in\{0.00,0.05,0.10, \ldots\} .
\end{aligned}
$$




$$
\begin{aligned}
& H(\theta)= \begin{cases}1: & \theta \geq \theta_{L}+1, \\
\theta-\theta_{L}: & \theta_{L} \leq \theta \leq \theta_{L}+1, \\
0: & \theta<\theta_{L},\end{cases} \\
& f(d)= \begin{cases}1: & d \leq \frac{r}{\theta_{L}+1}, \\
\frac{r}{d}-\theta_{L}: & \frac{r}{\theta_{L}+1} \leq d \leq \frac{r}{\theta_{L}}, \\
0: & d \geq \frac{r}{\theta_{L}} .\end{cases}
\end{aligned}
$$

Without loss of generality, $\mu$ is set as the reciprocal of the unit time and thus $\mu=1$. Thus, in a unit time one product is produced in average. From $\lambda=0.6$, the maximal availability of the process, which is a production rate when all orders from customers are accepted, is set as $\lambda / \mu=0.6$. From the setting of parameters, we have $d_{\max }=r / \theta_{L}=4$, and by (16) the expected utility of customers becomes

$$
\begin{aligned}
& u(\vec{d})=\sum_{i=-s}^{-1} p_{i}(\vec{d}) r+\sum_{i=0}^{I_{\max }} p_{i}(\vec{d}) \\
& \quad \cdot\left\{r\left(\theta_{i}-\theta_{L}\right)-\left(\frac{i+1}{\mu}\right)\left(\frac{\theta_{i}^{2}}{2}-\frac{\theta_{L}^{2}}{2}\right)\right\} .
\end{aligned}
$$

4.1. Optimal Quoted Lead Time. Optimal quoted lead time and the expected average profit for each $s$ are shown in Table 1 in the case that $c=0$ and $c=1$. The expected utilities of customers under the optimal policies are also given.

From Table 1, optimal quoted lead time is first increasing and then decreasing in $s$, which agrees with the result of Savaşaneril et al. [14], in which no delay opportunity cost is incurred; that is, $c=0$. Under the optimal policies, the values of $R(\vec{d}), S(\vec{d}), D(\vec{d})$, and $T(\vec{d})$ are also given in Table 1 . When $s$ is small, more customers must be accepted to increase system's profit and thus quoted lead time is small, and as a result the expected delay to quoted lead time $T(\vec{d})$ (and delay cost $D(\vec{d})$ for $c=1$ ) is large. When $s$ increases, both $R(\vec{d})$ and $S(\vec{d})$ increase. For large $s$, many customers can receive finished products immediately at their arrivals, and thus more customers enter the system, whereas there are more finished products in inventory and more inventory costs are needed. Since $\lambda=0.6$ and $R=10$ mean that the upper bound of $R(\vec{d})$ is 6 , for large $s$ almost arriving customers enter the system whereas more inventory costs are needed under optimal policies, and thus the optimal total profit of the system becomes small for large $s$. Compared with the case that $c=0$, the optimal policy is sensitive to delay when $c=1$, which leads to long quoted lead time. As a result, $T(\vec{d})$ and $R(\vec{d})$ are smaller compared when $c=0$.

The utilities are increasing in $s$, because the delay is smaller for more amounts of base stocks. The optimal base stock level maximizing the system's profit for $c=0$ is 1 whereas it is 2 for $c=1$. When $c$ increases, the probability that the delay to the quoted lead time happens is more important, and thus more amounts of base stocks are needed. Under optimal policies for each $s$, the profit decreases in $c$, but the expected utility increases. For large $c$ the delay probability needs to be decreased, and as a result the higher quoted lead time is set under the optimal policy, and thus customer's utility increases although the fraction that customers enter the system decreases.

Since $d_{\min }=r /\left(\theta_{L}+1\right)=0.8$, where $d_{\min }$ is the maximal value satisfying $f(d)=1$, for small state $i d_{i}=d_{\text {min }}$ because for all $d \leq d_{\text {min }}$ customers enter the system with probability 1.

4.2. Linear Lead Time Quotation Policy. As a heuristic policy, we consider a linear lead time quotation policy which takes quoted lead time proportional to $i+1$ for nonnegative state $i$. That is, there is a constant $\alpha$ which satisfies

$$
d_{i}=\alpha \frac{i+1}{\mu} \quad(\alpha>0) .
$$

This quotation policy is denoted by $d(s, \alpha)$. Note that when $\alpha<d_{\text {min }}$, for small $i$ such that $d_{\text {min }}>\alpha((i+1) / \mu)$ it is set as $d_{i}=d_{\min }$. For large $i$ such that $d_{\max } \leq \alpha((i+1) / \mu)$, the customer does not enter under the policy, and $d_{i}$ is set as $d_{\max }$. For example, under $d(s, 0.6) d_{0}=d_{\min }=0.8, d_{1}=1.2, d_{2}=$ $1.8, d_{3}=2.4, d_{4}=3.0, d_{5}=3.6$, and $d_{i}=d_{\max }=4.0$ for all $i \geq 6$.

Table 2 gives the profits and utilities for $s=0,1,2,3,4$ when $\alpha=0.6,0.8,1.0,1.2$ when $c=1$. Note that $\alpha<1$ implies that the expected actual lead time is greater than the lead time quoted to the customer. In fact, as shown in Table 2, when $\alpha$ increases, the profit decreases whereas the utility increases, because the higher quoted lead time implies less numbers of entering customers and less delay to the quoted lead time.

4.3. Nonlinear Policy. Here we consider nonlinear lead time quotation policies and compare them with linear quoted lead time policies. Considered quoted lead time policies are given in Table 3(a). The word "linear" is the linear lead time quotation policy $d(s, 0.6)$. The convex policy means the quoted lead time is convex in state $i$ from 1 to 5 , whereas the concave policy means that it is concave in state $i$ from 1 to 5 . The optimal policy in Table 3(a) represents the optimal lead time policy for $s=2$ when $c=1$.

The profits and utilities for these policies are given in Table 3(b) when $c=1$. Compared with linear lead time quotation policy, the convex policy has more profits and less utilities, and the concave policy has those vice versa. From these results, when the profit is more important, the convex type policy is better and when the utility has more weights the concave type policy is desirable. Note that the optimal lead time quotation policy has more profit and less utility in comparison with three heuristic policies.

4.4. Comparison. For the optimal policy and the other policies, the pairs (profit, utility) for $s=1,2,3,4$ are plotted in $(x, y)$-plane, which is shown in Figure 3 in the case where $c=1$. In this figure, $d^{*}(s)$ is an optimal policy for base stock level $s$, and $c c(s)$ and $\operatorname{cv}(s)$ are concave and convex policies for 
TABLE 1: Optimal policy.

(a) $c=0$

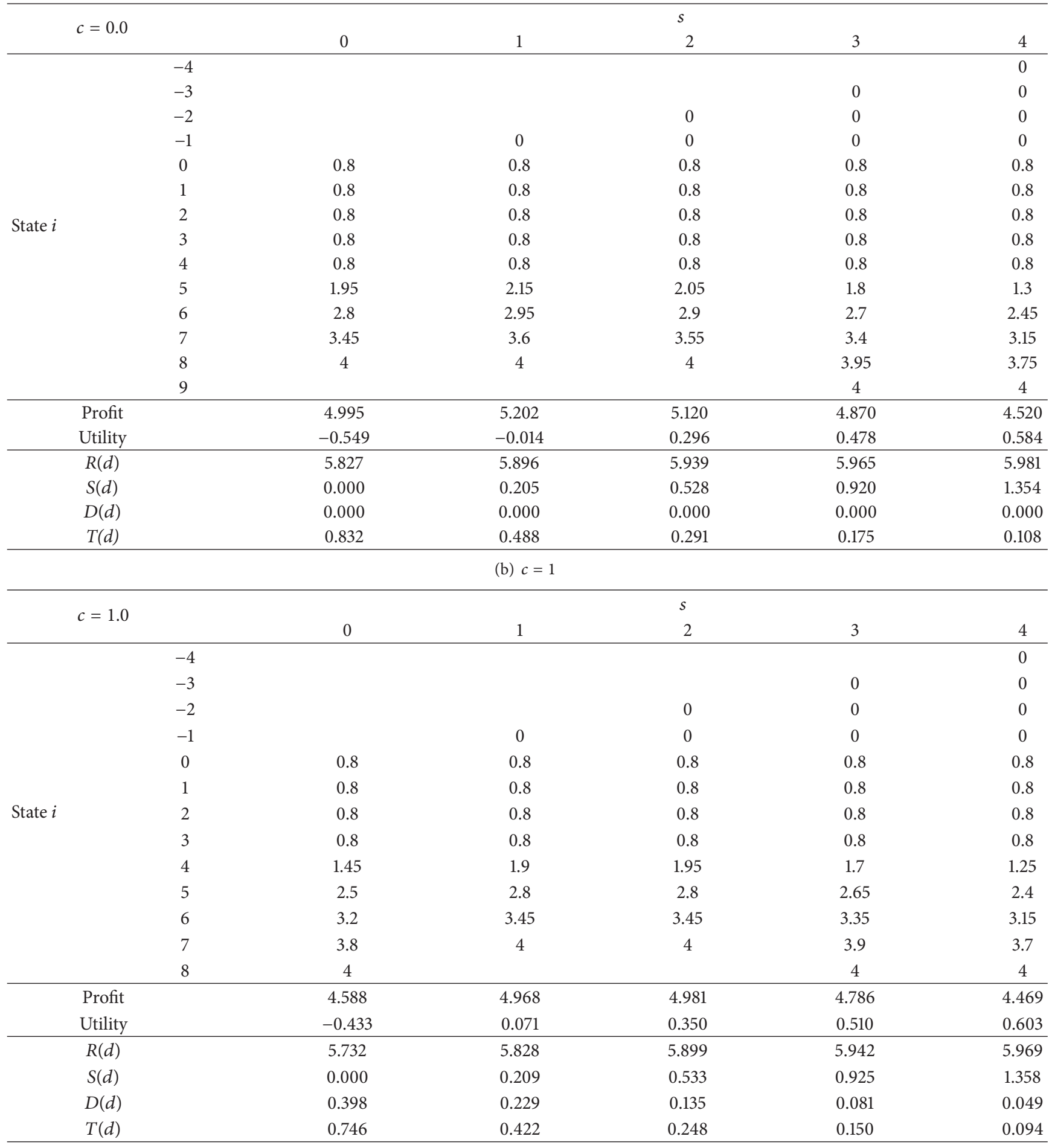

base stock $s$, defined in Section 4.3, respectively. The average profit and the expected utility for each policy are obtained by using stationary probabilities (5) in Section 2.3 and (13) and (16). For $s=1$, the profit of optimal policy is smaller than the optimal policy for $s=2$, and for any policy with $s=1$, the utility is also smaller than the optimal policy for $s=3$ or 4 . Thus, $s=1$ should not be selected. For $s=2$, the optimal policy maximizes the profit among all policies with all amounts of base stocks.

Compared with the optimal policy when $s=3$, the linear quoted lead time policies $d(2,0.6), d(2,0.8)$, and concave and convex policies $\mathrm{cv}(2)$ and $\operatorname{cs}(2)$ have more profits and 
TABLE 2: The linear lead time quotation policy.

\begin{tabular}{|c|c|c|c|c|c|c|}
\hline \multicolumn{2}{|c|}{$c=1.0$} & \multicolumn{5}{|c|}{ Base stock $s$} \\
\hline$\alpha$ & & 0 & 1 & 2 & 3 & 4 \\
\hline \multirow{2}{*}{0.6} & Profit & 4.078 & 4.751 & 4.861 & 4.708 & 4.415 \\
\hline & Utility & 0.111 & 0.413 & 0.562 & 0.642 & 0.686 \\
\hline \multirow{2}{*}{0.8} & Profit & 3.865 & 4.663 & 4.814 & 4.680 & 4.397 \\
\hline & Utility & 0.159 & 0.447 & 0.583 & 0.654 & 0.694 \\
\hline \multirow{2}{*}{1} & Profit & 3.163 & 4.385 & 4.671 & 4.596 & 4.345 \\
\hline & Utility & 0.204 & 0.489 & 0.611 & 0.672 & 0.705 \\
\hline \multirow{2}{*}{1.2} & Profit & 2.612 & 4.189 & 4.576 & 4.542 & 4.312 \\
\hline & Utility & 0.207 & 0.504 & 0.621 & 0.678 & 0.709 \\
\hline
\end{tabular}

TABLE 3: Comparison of heuristic policies.

(a) Policies

\begin{tabular}{lcccc}
\hline$i$ & Linear & Convex & Concave & Optimal \\
\hline 0 & 0.8 & 0.8 & 0.8 & 0.8 \\
1 & 1.2 & 1 & 1.4 & 0.8 \\
2 & 1.8 & 1.5 & 2.1 & 0.8 \\
3 & 2.4 & 2 & 2.8 & 0.8 \\
4 & 3 & 2.8 & 3.2 & 2.95 \\
5 & 3.6 & 3.6 & 3.6 & 3.45 \\
6 & 4 & 4 & 4 & 4 \\
7 & 4 & 4 & 4 & 4 \\
8 & 4 & 4 & 4 & 4 \\
\hline
\end{tabular}

(b) Profits and utilities

\begin{tabular}{|c|c|c|c|c|c|c|}
\hline \multirow{2}{*}{\multicolumn{2}{|c|}{$c=1$}} & \multicolumn{5}{|c|}{ Base stock $s$} \\
\hline & & 0 & 1 & 2 & 3 & 4 \\
\hline Linear & Profit & 4.078 & 4.751 & 4.861 & 4.708 & 4.415 \\
\hline$\alpha=0.6$ & Utility & 0.111 & 0.413 & 0.562 & 0.642 & 0.686 \\
\hline \multirow{2}{*}{ Convex } & Profit & 4.236 & 4.819 & 4.898 & 4.731 & 4.430 \\
\hline & Utility & 0.044 & 0.370 & 0.535 & 0.625 & 0.677 \\
\hline \multirow{2}{*}{ Concave } & Profit & 3.958 & 4.701 & 4.834 & 4.692 & 4.405 \\
\hline & Utility & 0.143 & 0.436 & 0.576 & 0.650 & 0.691 \\
\hline \multirow{2}{*}{ Optimal } & Profit & 4.588 & 4.968 & 4.981 & 4.786 & 4.469 \\
\hline & Utility & -0.433 & 0.071 & 0.350 & 0.510 & 0.603 \\
\hline
\end{tabular}

more utilities. Therefore, the optimal policy for $s=3$ is not needed to be used. Similar properties hold between the optimal policy for $s=4$ and several policies with $s=3$, and thus the optimal policy for $s=2$ will not be selected. The best policy depends on the thinking of decision maker.

\section{Conclusions}

In this paper, the base stock model with quoted lead time is considered and as performance measure the system profit and customer's utility are considered. It is formulated as a Markov chain when the lead time quotation policy is given, and it is also formulated as a Markov decision process to derive optimal policies for maximizing the system's profit.
Utility function of a customer is introduced and the heuristic policies for increasing utility of customers are proposed. The numerical results show that quoted lead time should be set by considering not only profit of the system but also the utility of customers. The optimal policy gives the smaller utilities, and in particular if the number of base stocks is not appropriate, then the other better policy exists on both profits and utilities than the optimal policy. It is noted that, throughout the numerical experiments, the base stock level is the most important factor for maximizing profits. That is, optimal base stock level and optimal policies are needed to be derived, but this optimal policy leads to poor expected utilities, and thus heuristic policies such as linear lead time policies should be considered to balance the system's profit and customer's expected utility. 


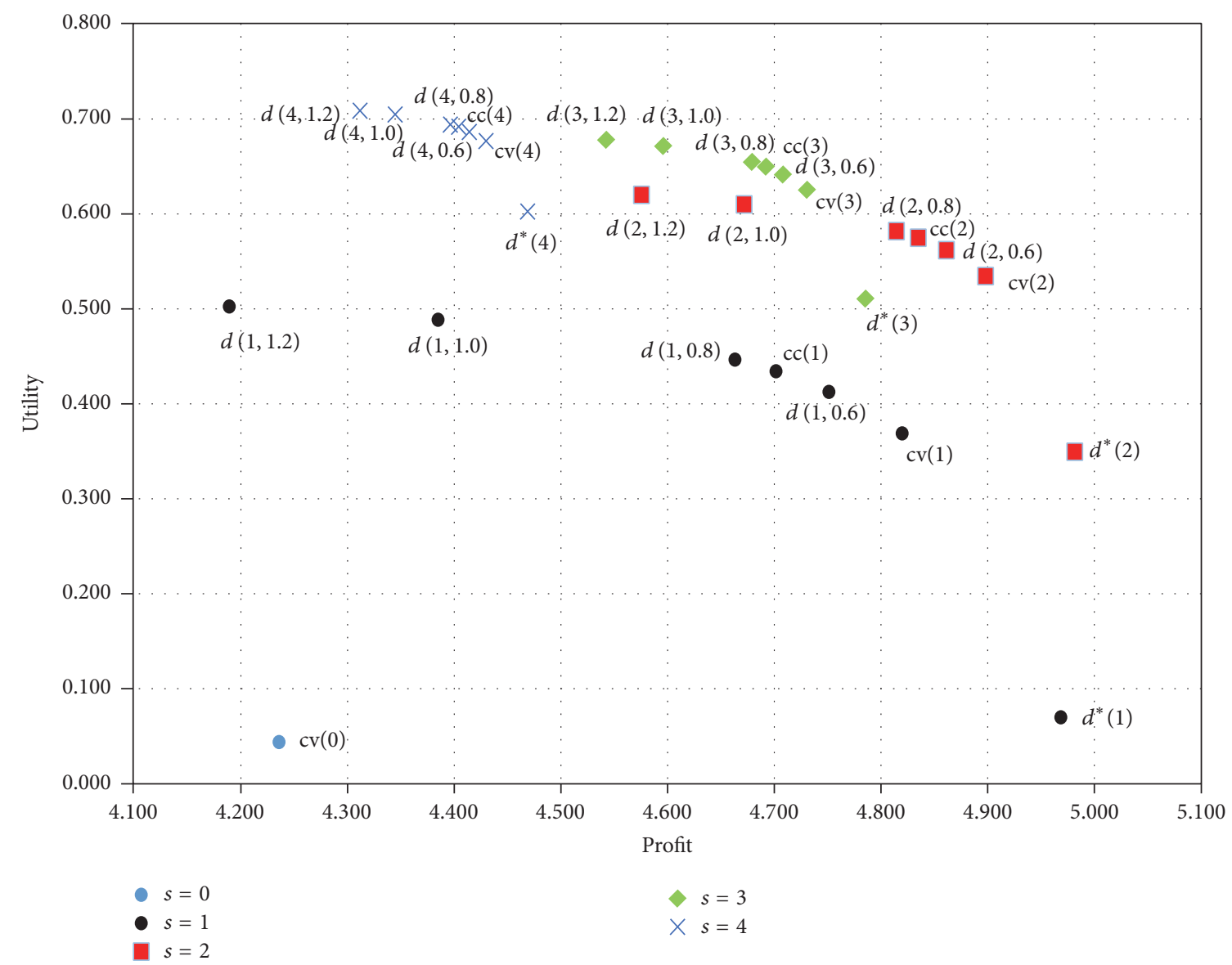

FIgURE 3: Comparison on profits and utilities.

Theoretical consideration on utilities under optimal policies and the other heuristic policies will be needed. In addition, the cases with general distributions of processing times and multistage manufacturing systems are also interesting. They are left for future research.

\section{Conflicts of Interest}

The authors declare that there are no conflicts of interest regarding the publication of this paper.

\section{Acknowledgments}

This paper is supported by JSPS Grant-in-Aid for Scientific Research (C) 16K01239.

\section{References}

[1] I. Duenyas and W. J. Hopp, "Quoting Customer Lead Times," Management Science, vol. 41, no. 1, pp. 43-57, 1995.

[2] B. Ata and T. L. Olsen, "Near-optimal dynamic lead-time quotation and scheduling under convex-concave customer delay costs," Operations Research, vol. 57, no. 3, pp. 753-768, 2009.
[3] R. Kapuscinski and S. Tayur, "Reliable due-date setting in a capacitated MTO system with two customer classes," Operations Research, vol. 55, no. 1, pp. 56-74, 2007.

[4] Z. Wu, B. Kazaz, S. Webster, and K.-K. Yang, "Ordering, pricing, and lead-time quotation under lead-time and demand uncertainty," Production and Operations Management, vol. 21, no. 3, pp. 576-589, 2012.

[5] B. Selçuk, "Adaptive lead time quotation in a pull production system with lead time responsive demand," Journal of Manufacturing Systems, vol. 32, no. 1, pp. 138-146, 2013.

[6] S. A. Slotnick, "Lead-time quotation when customers are sensitive to reputation," International Journal of Production Research, vol. 52, no. 3, pp. 713-726, 2014.

[7] A. B. Hafızoğlu, E. S. Gel, and P. Keskinocak, "Price and lead time quotation for contract and spot customers," Operations Research, vol. 64, no. 2, pp. 406-415, 2016.

[8] X. Zhao, K. E. Stecke, and A. Prasad, "Lead time and price quotation mode selection: Uniform or differentiated?" Production and Operations Management, vol. 21, no. 1, pp. 177-193, 2012.

[9] P. Keskinocak, R. Ravi, and S. Tayur, "Scheduling and reliable lead-time quotation for orders with availability intervals and lead-time sensitive revenues," Management Science, vol. 47, no. 2, pp. 264-279, 2001.

[10] B. Ata, "Dynamic control of a multiclass queue with thin arrival streams," Operations Research, vol. 54, no. 5, pp. 876-892, 2006. 
[11] K. Charnsirisakskul, P. M. Griffin, and P. n. Keskinocak, "Pricing and scheduling decisions with leadtime flexibility," European Journal of Operational Research, vol. 171, no. 1, pp. 153169,2006

[12] S. K. Chaharsooghi, M. Honarvar, M. Modarres, and I. N. Kamalabadi, "Developing a two stage stochastic programming model of the price and lead-time decision problem in the multiclass make-to-order firm," Computers and Industrial Engineering, vol. 61, no. 4, pp. 1086-1097, 2011.

[13] T. Xiao, J. Jin, G. Chen, J. Shi, and M. Xie, “Ordering, wholesale pricing and lead-time decisions in a three-stage supply chain under demand uncertainty," Computers \& Industrial Engineering, vol. 59, no. 4, pp. 840-852, 2010.

[14] S. Savaşaneril, P. M. Griffin, and P. n. Keskinocak, "Dynamic lead-time quotation for an $M / M / 1$ base-stock inventory queue," Operations Research, vol. 58, no. 2, pp. 383-395, 2010.

[15] P. Guo and P. Zipkin, "Analysis and comparison of queues with different levels of delay information," Management Science, vol. 53, no. 6, pp. 962-970, 2007.

[16] P. Guo and P. Zipkin, "The effects of information on a queue with balking and phase-type service times," Naval Research Logistics, vol. 55, no. 5, pp. 406-411, 2008.

[17] K. Nakade and H. Niwa, "Lead time quotation and customer utility in an M/M type base stock system," Proceedings of MIM, IFAC Paper Online, vol. 49, no. 12, pp. 1520-1525, 2016.

[18] M. A. Pinsky and S. Karlin, An Introduction to Stochastic Modeling, Academic Press, 4th edition, 2011.

[19] M. L. Puterman, Decision Markov Processes; Discrete Stochastic Dynamic Programming, Wiley, 1994. 


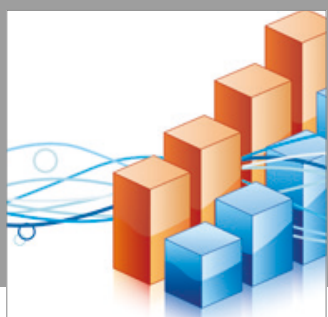

Advances in

Operations Research

vatersals

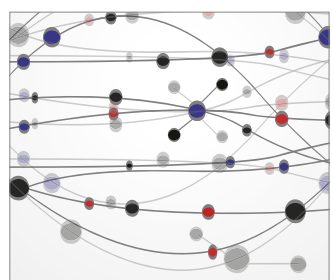

\section{The Scientific} World Journal
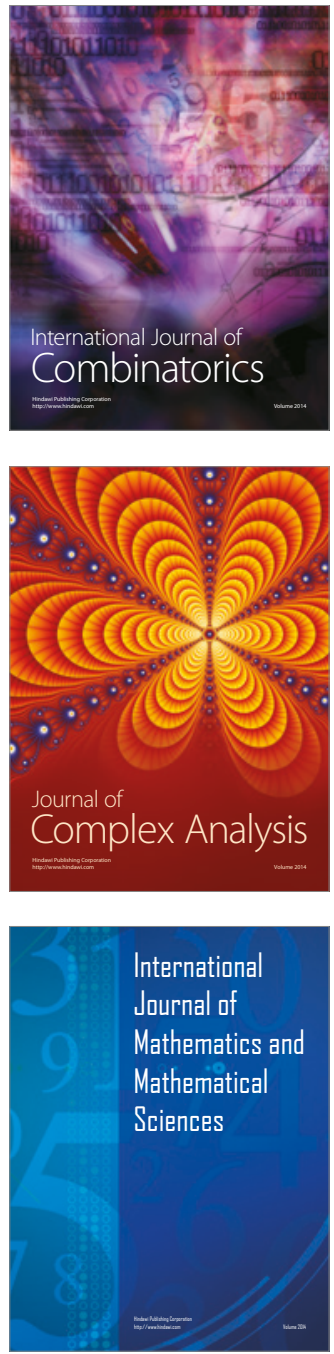
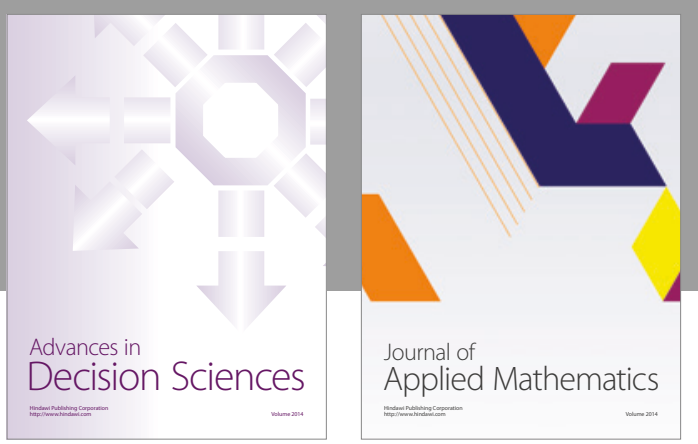

Algebra

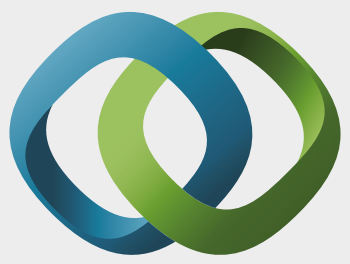

\section{Hindawi}

Submit your manuscripts at

https://www.hindawi.com
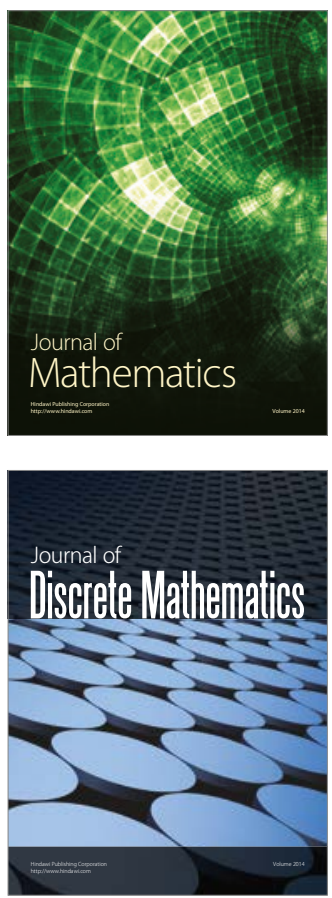

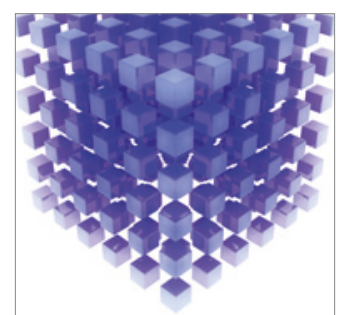

Mathematical Problems in Engineering
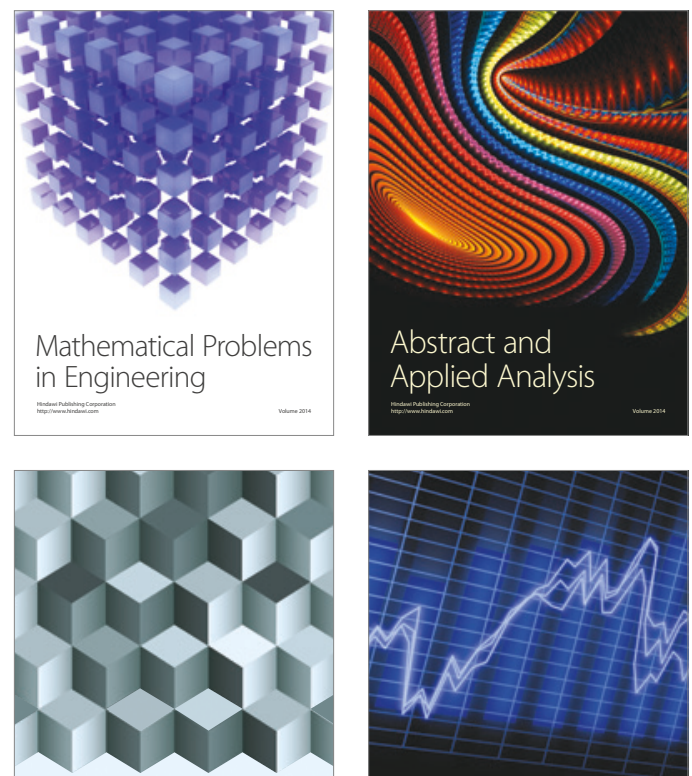

Journal of

Function Spaces

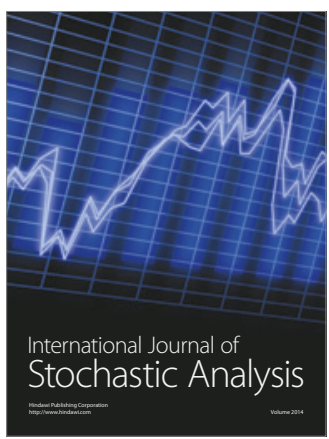

Probability and Statistics
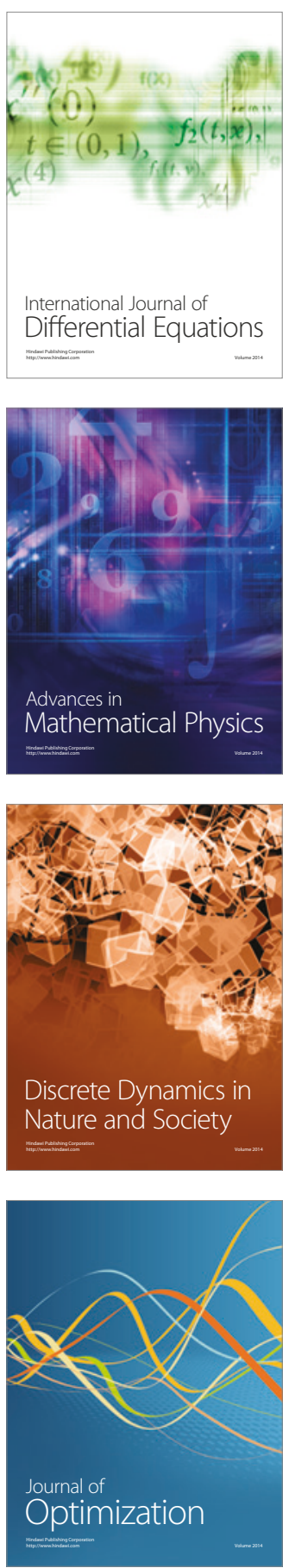